hep-th/0208066

MCTP-02-41

PUPT-2046

\title{
Strings in RR Plane Wave Background at Finite Temperature
}

\author{
Leopoldo A. Pando Zayas ${ }^{1}$ and Diana Vaman ${ }^{2}$ \\ ${ }^{1}$ Michigan Center for Theoretical Physics \\ Randall Laboratory of Physics, The University of Michigan \\ Ann Arbor, MI 48109-1120 \\ ${ }^{1}$ School of Natural Sciences \\ Institute for Advanced Study \\ Princeton, NJ 08540 \\ ${ }^{2}$ Department of Physics \\ Princeton University \\ Princeton, NJ 08544
}

\begin{abstract}
We calculate the thermal partition function in the canonical ensemble for type IIB superstrings in the plane wave background with constant null R-R 5-form. The Hagedorn temperature is found to be higher than the corresponding value for strings in flat space. In the limit corresponding to the weakly coupled field theory we find that the Hagedorn temperature is pushed to infinity. The key property of strings in the plane wave background under investigation on which our result relies is that the effective mass of the bosonic and fermionic coordinates in the light-cone gauge is proportional to the momentum $p^{+}$. The free energy is finite as the Hagedorn temperature is approached from below, suggesting a possible phase transition.
\end{abstract}

email addresses: lpandoz@ias.edu, dvaman@feynman.princeton.edu 


\section{Introduction and Summary}

The AdS/CFT correspondence has given a concrete realization of the connection between gauge theories and string theory in the context of $\mathcal{N}=4$ supersymmetric Yang-Mills and type IIB string theory on $A d S_{5} \times S^{5}$ [1]. One of the immediate implications of the correspondence, pointed out by Witten in [2], is the relation between the corresponding theories at nonzero temperature. Namely, it was conjectured that a direct relation exists between a nonzero temperature field theory and the properly thermalized supergravity background. This correspondence was effectively anticipated in the series of papers [3]. A remarkable feature of the nonzero temperature correspondence is that it involves nonsupersymmetric theories on both sides and it is therefore a more dynamical connection than the usual zero-temperature correspondence.

Recently, Berenstein, Maldacena and Nastase (BMN) [4] put a new twist on the AdS/CFT correspondence and proposed a gauge theory interpretation of the Penrose-Güven limit of $A d S_{5} \times S^{5}$ [5]. The IIB maximally supersymmetric plane wave background with constant null RR five form resulting from the limit is

$$
\begin{aligned}
d s^{2} & =-2 d x^{+} d x^{-}-\mu^{2} x_{i} x^{i}\left(d x^{+}\right)^{2}+d x_{i} d x^{i}, \\
F_{+1234} & =F_{+5678}=2 \mu .
\end{aligned}
$$

In this limit a particular sector of $\mathcal{N}=4 \mathrm{SYM}$ survives in the gauge theory side while the string theory is solvable in the light-cone gauge $[6,7]$. One particularly interesting observation that follows from the study of BMN operators is that certain quantities, such as the dimensions of the BMN operators, when calculated using perturbative gauge theory in the effective 't Hooft coupling $\left(\lambda^{\prime}\right)$ match precisely the string theory prediction which is reliable at strong coupling [8]. This provides an example of smooth interpolation between the weak and strong coupling regimes of a sector of $\mathcal{N}=4$ SYM. Additional exploration of more complicated observables was presented in [9].

In the context of the Penrose limit of $A d S_{5} \times S^{5}$, it is very natural to pose a similar question about the nonzero temperature correspondence. The present situation is very peculiar however. An important ingredient introduced in the analysis of [2] is the consideration of all backgrounds with the same asymptotic behavior. In the concrete case of $\mathcal{N}=4 \mathrm{SYM}$ this implies the need to consider at least thermalized $A d S_{5}$ and the Schwarzschild-AdS black hole. The inclusion of these two backgrounds implies the presence of Hawking-Page phase transitions. The question of the proper thermalization of the plane wave background is ambiguous. Moreover, the Penrose limit of the Schwarzschild-AdS black hole does not seem to naturally determine a horizon and therefore it lacks the notion of temperature [10]. Thus, the supergravity approach seems to be at least not straightforward. On the other hand, the fact that string theory on the plane wave can be quantized in the light-cone gauge gives us the possibility of exploring some of the corresponding thermodynamic properties.

Strings in flat space exhibit a Hagedorn density of states $\rho(M)=M^{-a} \exp (b M)$, with $a$ and $b$ constants characterizing the various string models. Given the exponential growth in the density of states, the theory cannot be defined above a critical temperature $T_{H} \sim 1 /\left(l_{s} b\right)$. This behavior has inspired a lot of speculations; one analogy that is frequently made is between the Hagedorn transition of strings and the confinement/deconfinement phase transition of QCD. 
It is natural to return to this question within the context of the BMN limit of $\mathcal{N}=4 \mathrm{SYM}$ since it provides an example of gauge/string relation on one side of which we have an exactly solvable string theory.

At first sight it seems very plausible that the high-temperature observables of string theory in the plane wave background have to be essentially the same as in flat space. An intuitive way to think about this is by using the fact that ultimately the main contribution to high temperature effects comes from the behavior of highly excited strings. Adding a mass term $(m)$ to the bosons does not seem to be a strong enough modification since, for any $m$ we have that for large enough excitation levels $(n)$, the expression for the frequencies $\sqrt{n^{2}+m^{2}}$ will be better and better approximated by the flat space expression: $n$. However, a key property of strings in the plane-wave background that invalidates the above reasoning is that in the lightcone gauge the mass parameter is not constant, but $p^{+}$dependent: $m=2 \pi \alpha^{\prime} \mu p^{+}$. We will show that this explicit dependence on $p^{+}$when properly taken into consideration modifies the modular properties of the partition function in a crucial way and in particular is responsible for the Hagedorn temperature being higher than the corresponding value for strings in flat space.

To make the connection to the BMN sector more precise recall the following relations between the string and the gauge theory quantities $\mu \alpha^{\prime} p^{+}=\frac{J}{\sqrt{\lambda}}, \frac{2 p^{-}}{\mu}=\Delta-J$, where $J$ is the R charge, $\Delta$ is the conformal dimension and $\lambda$ is the 't Hooft coupling. The BMN sector is singled out by considering operators with fixed $p^{+}$and finite $p^{-}$. Having fixed $p^{+}$means considering operators whose $\mathrm{R}$ charge J grows as $\sqrt{N}$ as $N \rightarrow \infty$. We, by integrating over $p^{+}$, are forced to effectively consider a larger sector than the BMN sector. This fact obscures the direct relevance of our string calculation for the gauge theory side.

Leaving aside the usual difficulties associated with defining thermodynamics in gravitational backgrounds, in this paper we compute the thermal partition function of the IIB superstring in the plane wave background with constant null R-R 5-form. Moreover, we restrict ourselves to the leading genus one contribution to the thermal partition function. We pay special attention to the Hagedorn temperature which has now a parametric dependence on $\mu$. We find that for $\mu \neq 0$ the Hagedorn temperature is higher than the corresponding value in flat space

$$
-\frac{\beta_{H}^{2}}{2 \pi \alpha^{\prime}}-16 \pi \gamma_{0}\left(\mu \beta_{H}\right)+16 \pi \gamma_{1 / 2}\left(\mu \beta_{H}\right)=0
$$

where $\gamma_{0}$ and $\gamma_{1 / 2}$ are the zero-point energies of integer and half-integer moded massive oscillators respectively. In the limit $\mu l_{s} \rightarrow 0$, the first correction to the flat space value of the Hagedorn temperature is $T_{H} \approx 1 / 2 \pi l_{s}+4 \mu$. In the "weak field theory limit" $\left(\mu l_{s} \rightarrow \infty\right)$ we find that the Hagedorn temperature is pushed to infinity. We also provide an approximate expression for the free energy near the Hagedorn temperature assuming that the main contribution comes from the one-string sector. A saddle point evaluation shows that the free energy and is finite as we approach the Hagedorn temperature from below. Therefore a phase transition could take place at $T_{H}=1 / \beta_{H}$. Given that in the canonical ensemble the energy fluctuations are notoriously large nearby $T_{H}$, the answer to the true nature (limiting or phase transition) of the Hagedorn temperature is hold by a complementary picture to the one presented here, namely by the microcanonical ensemble.

The paper is organized as follows. Section 2 contains a short review of the canonical en- 
semble for strings in the light-cone gauge. Section 3 contains the essential ingredients in the calculation of the thermal partition function for the Green-Schwarz (GS) strings in the plane wave background. Throughout section 3 we make explicit how our results generalize those for GS strings in flat space. In section 4 we assemble all the ingredients and discuss the two natural limiting cases: flat space $\left(\mu l_{s} \rightarrow 0\right)$ and the weak field theory $\left(\mu l_{s} \rightarrow \infty\right)$ and discuss the thermodynamic quantities of interest (free energy and specific heat).

\section{Review of the canonical ensemble of strings in light- cone}

Since in the following sections we need to address the thermal properties of strings whose action is known only in the light-cone gauge-fixed form, and therefore a covariant treatment is not available, we begin with a brief review of the canonical ensemble of light-cone strings $[11,12]$. As explained in [11], the finite temperature one-string partition function is defined by

$$
Z_{1}(\beta)=\operatorname{Tr} \mathrm{e}^{-\beta \mathrm{P}^{0}}=\operatorname{Tr} \mathrm{e}^{-\beta\left(\mathrm{P}^{+}+\mathrm{P}^{-}\right)}
$$

where $\beta$ is the inverse temperature, $P^{+}\left|\Phi>=p^{+}\right| \Phi>$ is the light-cone momentum, and

$P^{-} \mid \Phi>=\frac{1}{p^{+}}\left(H_{l c} \mid \Phi>\right)$ with $H_{l c}$ being the light-cone Hamiltonian. Thus the partition function can be written as

$$
Z_{1}(\beta)=l_{s} \int d p^{+} e^{-\beta p^{+}} z_{l c}\left(\beta / p_{+}\right)
$$

For open strings, the partition function is evaluated over a cylindrical worldsheet, with periodic time $t \sim t+\beta / p^{+}$, while for closed strings we must additionally impose the level-matching constraint: equal momentum carried by the left and right oscillators. This is usually done by means of a Lagrange multiplier

$$
Z_{1}(\beta)=l_{s} \int d p^{+} \int d \lambda e^{-\beta p^{+}} \operatorname{Tr} e^{-\frac{\beta}{p^{+}} H_{l c}+2 \pi i \lambda\left(N_{L}-N_{R}\right)} .
$$

From the point of view of the light-cone partition function, the effect of the angle $\lambda$ is to twist the ends of the cylinder before identifying them.

Another useful way to approach the partition function is by way of the path integral formalism, with the light-cone partition function given by

$$
z_{l c}=\int \mathcal{D} X e^{-\int_{T} d^{2} z L[X(z)]}
$$

and where the worldsheet action is integrated over a torus $T$ with modular parameter

$$
\tau=\tau_{1}+i \tau_{2}=\lambda+i \frac{\beta}{2 \pi p^{+}} .
$$

One of the advantages of the path integral approach is that it makes the modular properties of the partition function manifest. We will repeatedly exploit this fact in the following sections. 
Having defined the one-string partition function it is natural to define the partition function for a gas of strings at constant temperature (canonical ensemble). The partition function for a gas of strings is built out of the one-string partition function through further exponentiation

$$
\ln Z(\beta)=\frac{1}{2} \sum_{r=1}^{\infty}\left[1-(-1)^{r}\right] \frac{Z_{1}(\beta r)}{r},
$$

for the supersymmetric case and $\ln Z(\beta)=\frac{1}{2} \sum_{r=1}^{\infty} \frac{1}{r} Z_{1}(\beta r)$ for the bosonic case. The thermodynamical potential associated with the canonical ensemble is the free energy

$$
F(\beta)=-\frac{1}{\beta} \ln Z(\beta) .
$$

Another thermodynamical quantity of interest is the specific heat

$$
c_{V}=\beta^{2} \frac{\partial^{2}}{\partial \beta^{2}} \ln Z(\beta) .
$$

At temperatures higher than the Hagedorn temperature $T_{H}$ the free energy diverges. If the free energy remains finite while $T_{H}$ is approached from below there is the possibility of a phase transition. On the other hand, if the free energy and specific heat are infinite, the Hagedorn temperature is a limiting (maximal) temperature.

\section{Partition function of GS strings in the pp-wave back- ground}

The most straightforward way to obtain the partition function is through the Hamiltonian. The light-cone Hamiltonian of the first-quantized GS IIB string in the plane wave background with constant null R-R five-form background is given by $[6,7]$ :

$$
\begin{aligned}
H_{l c} & =\frac{1}{\alpha^{\prime}}\left[m\left(a_{0}^{i \dagger} a_{0}^{i}+S_{0}^{i \dagger} S_{0}^{i}\right)\right. \\
& \left.+\sum_{n=1}^{\infty} \sqrt{n^{2}+m^{2}}\left(\sum_{i=1}^{8}\left(a_{n}^{i \dagger} a_{n}^{i}+\tilde{a}_{n}^{i \dagger} \tilde{a}_{n}^{i}\right)+\sum_{a=1}^{8}\left(S_{n}^{a \dagger} S_{n}^{a}+\tilde{S}_{n}^{a \dagger} \tilde{S}_{n}^{a}\right)\right)\right]
\end{aligned}
$$

where we follow the conventions of Metsaev and Tseytlin [7]. We want to emphasize at this point that the mass parameter is $p^{+}$-dependent:

$$
m=\mu\left(2 \pi \alpha^{\prime}\right) p^{+} .
$$

Explicitly performing the trace of the light-cone Hamiltonian, and implementing the levelmatching constraint $\lambda\left(N_{R}-N_{L}\right)$ as discussed in the previous section leads to the light-cone 
partition function

$$
\begin{aligned}
z_{l c}\left(\frac{\beta}{p^{+}}, \lambda\right)= & \prod_{n, n^{\prime}=1}^{\infty}\left[\exp \sum_{N, N^{\prime}=0}^{\infty}\left(-\frac{\beta}{\alpha^{\prime} p^{+}} N \sqrt{n^{2}+m^{2}}-\frac{\beta}{\alpha^{\prime} p^{+}} N^{\prime} \sqrt{n^{\prime 2}+m^{2}}+2 \pi i \lambda\left(N n-N^{\prime} n^{\prime}\right)\right)\right]^{8} \\
& {\left[\exp \sum_{N, N^{\prime}=0,1}\left(-\frac{\beta}{\alpha^{\prime} p^{+}} N \sqrt{n^{2}+m^{2}}-\frac{\beta}{\alpha^{\prime} p^{+}} N^{\prime} \sqrt{n^{\prime 2}+m^{2}}+2 \pi i \lambda\left(N n-N^{\prime} n^{\prime}\right)\right)\right]^{8} } \\
& {\left[\exp \sum_{N=0}^{\infty}\left(-\frac{\beta}{\alpha^{\prime} p^{+}} N m\right)\right]^{8}\left[\exp \sum_{N=0,1}\left(-\frac{\beta}{\alpha^{\prime} p^{+}} N m\right)\right]^{8} } \\
= & \prod_{n=1}^{\infty}\left|\left(\frac{1+\exp \left(-\frac{\beta}{\alpha^{\prime} p^{+}} \sqrt{n^{2}+m^{2}}+2 \pi i \lambda n\right)}{1-\exp \left(-\frac{\beta}{\alpha^{\prime} p^{+}} \sqrt{n^{2}+m^{2}}+2 \pi i \lambda n\right)}\right)^{8}\right|^{2}\left(\frac{1+\exp \left(-\frac{\beta}{\alpha^{\prime} p^{+}} m\right)}{1-\exp \left(-\frac{\beta}{\alpha^{\prime} p^{+}} m\right)}\right)^{8} \\
= & \prod_{n \in \mathbf{Z}}\left(\frac{1+\exp \left(-\frac{\beta}{\alpha^{\prime} p^{+}} \sqrt{n^{2}+m^{2}}+2 \pi i \lambda n\right)}{1-\exp \left(-\frac{\beta}{\alpha^{\prime} p^{+}} \sqrt{n^{2}+m^{2}}+2 \pi i \lambda n\right)}\right)^{8},
\end{aligned}
$$

where we have taken into account that the usual normal ordering shift cancels between fermions and bosons (in each sector it equals the zero-point energy $m / 2+\sum_{n=1}^{\infty} \sqrt{n^{2}+m^{2}}$ ).

The above expression is formally the answer for the partition function. However, it is very difficult to extract information directly in this form. We turn to the path integral approach where we can derive the modular properties of partition function. The latter will enable us to study the thermodynamic properties of the strings.

\subsection{Scalars}

To motivate some of the further manipulations needed to extract information from the partition function presented above we will also include the treatment for flat space. Another important point we would like to make is that by considering strings at nonzero temperature we explicitly break supersymmetry and therefore some of the thermodynamic properties of strings can be seen equally well by considering only the bosonic sector. Thus, we analyze first the bosonic contribution to the free energy, keeping in mind similarities with a system of closed bosonic strings. Let us briefly recall the determination of the Hagedorn temperature for the bosonic string in flat space. Each scalar degree of freedom contributes the following factor to the the transverse partition function

$$
\begin{aligned}
z_{l c}^{(0,0)}\left(\frac{\beta}{p^{+}}, \lambda\right) & =\int_{-\infty}^{\infty} d p \exp \left(-\frac{\beta p^{2}}{2 \alpha^{\prime} p^{+}}\right) \exp \left(\frac{\beta}{\alpha^{\prime} p^{+}} \sum_{n=1}^{\infty} n\right) \prod_{n=1}^{\infty}\left|\frac{1}{1-\exp \left(-\frac{\beta}{\alpha^{\prime} p^{+}} n+2 \pi i \lambda n\right)}\right|^{2} \\
& =2 \sqrt{\frac{\pi \alpha^{\prime} p^{+}}{\beta}} \exp \left(-\frac{1}{12} \frac{\beta}{\alpha^{\prime} p^{+}}\right) \prod_{n=1}^{\infty}\left|\frac{1}{1-\exp \left(-\frac{\beta}{\alpha^{\prime} p^{+}} n+2 \pi i \lambda n\right)}\right|^{2}
\end{aligned}
$$

A convenient way of rewriting the above expression is by means of introducing a complex variable

$$
q=\exp (2 \pi i \tau) \quad, \quad \tau=\lambda+i \frac{\beta}{2 \pi \alpha^{\prime} p^{+}} .
$$


In this variable the partition function takes the following simple form which highlights its modular properties

$$
z_{l c}^{(0,0)}(\tau)=\tau_{2}^{-1 / 2}\left((q \bar{q})^{\frac{1}{24}}\left|\prod_{n=1}^{\infty}\left(1-q^{n}\right)\right|\right)^{-2}=\tau_{2}^{-1 / 2}|\eta(\tau)|^{-2} .
$$

For $d-2$ transverse scalars, the one-string partition function reads

$$
\begin{aligned}
Z_{1}^{(0,0)}(\beta) & =l_{s} \int_{0}^{\infty} d p^{+} \int_{0}^{1} d \lambda \exp \left(-\beta p^{+}\right)\left(\sqrt{\frac{2 \pi \alpha^{\prime} p^{+}}{\beta}}\left|\eta\left(\lambda+i \frac{\beta}{2 \pi p^{+}}\right)\right|^{-2}\right)^{d-2} \\
& =\frac{\beta}{2 \pi l_{s}} \int d \tau_{1} \int_{0}^{\infty} d \tau_{2} \exp \left(-\frac{\beta^{2}}{2 \pi \alpha^{\prime} \tau_{2}}\right) 2^{d-2} \tau_{2}^{-\frac{d-2}{2}+2}|\eta(\tau)|^{-2(d-2)}
\end{aligned}
$$

where we have made the change of variable $\frac{\beta}{2 \pi \alpha^{\prime} p^{+}}=\tau_{2}$. The UV asymptotic behavior, $\tau_{2} \rightarrow 0$, of the one-string partition function is uncovered by using the modular properties of the Dedekind $\eta(\tau)$-function. Under the $S$-modular transformation $\tau \rightarrow-1 / \tau$

$$
\eta\left(-\frac{1}{\tau}\right)=\sqrt{-i \tau} \eta(\tau) .
$$

Substituting (3.8) into (3.7) one finds that the integrand of the one-string partition function in the UV regime behaves as

$$
\tau_{2}^{-\frac{d+2}{2}}|\tau|^{d-2} \exp \left(4 \pi \tau_{2} \frac{d-2}{24|\tau|^{2}}-\frac{\beta^{2}}{2 \pi \alpha^{\prime} \tau_{2}}\right) .
$$

At temperatures higher than the Hagedorn temperature

$$
\beta_{H}=\frac{1}{T_{H}}=\sqrt{\frac{\pi^{2}(d-2)}{3}} l_{s}
$$

the free energy diverges.

To evaluate the contribution of the scalar modes of the IIB GS superstring in the plane wave background we choose to do a path integral calculation. This approach will pay off in the sense that the modular properties of the partition function are much more transparent when the partition function is expressed as a double product. Let us therefore begin with

$$
z_{l c}^{(0,0)}(\tau, m)=\int \mathcal{D} X \exp \left[-\int_{T} d^{2} z \bar{X}\left(-\partial_{z} \partial_{\bar{z}}+m^{2}\right) X\right]
$$

where the worldsheet integral is taken over a torus: $z=\xi_{1}+\tau \xi_{2}$, and the modular parameter is denoted as usual by $\tau$. Substituting the Fourier decomposition of the doubly periodic function

$$
X\left(\xi_{1}, \xi_{2}\right)=\sum_{n 1, n 2 \in \mathbf{Z}} X_{n 1, n 2} \exp \left[2 \pi i\left(n_{1} \xi_{1}+n_{2} \xi_{2}\right)\right]
$$


in the path integral and using

$$
\begin{aligned}
d^{2} z & =d \xi_{1} d \xi_{2} \tau_{2} \\
\partial_{z} \partial_{\bar{z}} & =\frac{1}{4 \tau_{2}{ }^{2}}\left(|\tau|^{2} \partial_{1}{ }^{2}-2 \tau_{1} \partial_{1} \partial_{2}+\partial_{2}{ }^{2}\right)
\end{aligned}
$$

we can explicitly perform the Gaussian integrals over $X_{n 1, n 2}$ in (3.11)

$$
z_{l c}^{(0,0)}(\tau, \mu)=\left[\prod_{n_{1}, n_{2} \in \mathbf{Z}} \tau_{2}\left(\left(\frac{2 \pi}{4 \tau_{2}}\right)^{2}\left|n_{1} \tau-n_{2}\right|^{2}+m^{2}\right)\right]^{-(d-2)} .
$$

The double product form of the partition function makes manifest its modular properties. For example, recalling that $m$ depends explicitly on $p^{+}=\frac{\beta}{2 \pi \alpha^{\prime} \tau_{2}}$ and substituting $m=2 \pi \alpha^{\prime} \mu p^{+}$ into (3.15) one derives:

$$
\left.z_{l c}^{(0,0)}(-1 / \tau, \mu /|\tau|)\right)=z_{l c}^{(0,0)}(\tau, \mu)
$$

For cases considered in the literature $[13,14], m$ was uncorrelated with the torus modular parameter, and (3.16) is then replaced by $z_{l c}(-1 / \tau, m|\tau|)=z_{l c}(\tau, m)^{1}$.

Following [15], one of the infinite products in (3.15) can be performed and the result is

$$
\begin{aligned}
z_{l c}^{(0,0)}(\tau, m)= & \exp \left[-2 \pi(d-2) \tau_{2}\left(m / 2+\sum_{n=1}^{\infty} \sqrt{n^{2}+m^{2}}\right)\right] \\
& {\left[\prod_{n \in \mathbf{Z}}\left(1-\exp \left[2 \pi\left(-\tau_{2} \sqrt{n^{2}+m^{2}}+i \tau_{1} n\right)\right]\right)\right]^{-(d-2)} }
\end{aligned}
$$

This expression is in fact a natural generalization of the Dedekind $\eta$ function, where the first exponent substitutes the factor $q^{1 / 24}$ of the $\eta$-function. We use $\zeta$-function regularization to define the Casimir energy of a system of massive oscillators

$$
\begin{aligned}
\gamma_{0}(m) & =\frac{m}{2}+\sum_{n=1}^{\infty} \sqrt{n^{2}+m^{2}} \\
& =\frac{m}{2}+\left[-\frac{1}{12}+\frac{1}{2} m-\frac{1}{2} m^{2} \ln \left(4 \pi e^{-\gamma}\right)+\sum_{n=2}^{\infty}(-1)^{n} \frac{\Gamma\left(n-\frac{1}{2}\right)}{n ! \Gamma\left(-\frac{1}{2}\right)} \zeta(2 n-1) m^{2 n}\right]
\end{aligned}
$$

\footnotetext{
${ }^{1}$ After trivial relabellings in the double product,

$$
z_{l c}^{(0,0)}(-1 / \tau, \mu /|\tau|)=\left[\prod_{n_{1}, n_{2} \in \mathbf{Z}} \frac{\tau_{2}}{|\tau|^{2}}\left(\left(\frac{2 \pi|\tau|^{2}}{4 \tau_{2}}\right)^{2} \frac{\left|-n_{1}-n_{2} \tau\right|^{2}}{|\tau|^{2}}+\left(\frac{\beta \mu|\tau|^{2}}{|\tau| \tau_{2}}\right)^{2}\right)\right]^{-(d-2)}=z_{l c}^{(0,0)}(\tau, \mu)
$$
}

whereas for constant $m$ we have

$$
z_{l c}^{(0,0)}(-1 / \tau, m|\tau|)=\left[\prod_{n_{1}, n_{2} \in \mathbf{Z}} \frac{\tau_{2}}{|\tau|^{2}}\left(\left(\frac{2 \pi|\tau|^{2}}{4 \tau_{2}}\right)^{2} \frac{\left|-n_{1}-n_{2} \tau\right|^{2}}{|\tau|^{2}}+m^{2}|\tau|^{2}\right)\right]^{-(d-2)}=z_{l c}^{(0,0)}(\tau, m)
$$


where $\gamma$ is the Euler constant. Note that the zero-th order term in the $\gamma_{0}(m)$ mass expansion is the same as for a massless scalar $\sum_{n=1}^{\infty} n=\zeta(-1)=-1 / 12$. The terms of order greater and equal to 2 can be derived as follows:

$$
\begin{aligned}
\sum_{n=1}^{\infty} \sqrt{n^{2}+m^{2}} & =\sum_{n=1}^{\infty} \frac{1}{\Gamma(-1 / 2)} \int_{0}^{\infty} d t \exp \left(-t\left(n^{2}+m^{2}\right)\right) t^{-3 / 2} \\
& =\frac{1}{2 \Gamma(-1 / 2)} \int_{0}^{\infty} d t\left[\Theta_{3}\left(0, \frac{i t}{\pi}\right)-1\right] \exp \left(-t m^{2}\right) t^{-3 / 2}
\end{aligned}
$$

and after Taylor expanding the exponential, use further that

$$
\int_{0}^{\infty} d x x^{s-1}\left[\Theta_{3}\left(0, i x^{2}\right)-1\right]=\pi^{-s / 2} \Gamma\left(\frac{s}{2}\right) \zeta(s), \quad s \geq 2 .
$$

The expression obtained in (3.17) for the partition function also represents the generalization to the complex plane of the modular forms $f_{1}^{(m)}\left(e^{-2 \pi \tau_{2}}\right)$ introduced by [13]. The feature that makes this generalization special is that the appropriate modular form for massive transverse scalars (as opposed to massless transverse scalars) is not a holomorphic function of the complex torus parameter $\tau$.

The UV behavior of the scalar degrees of freedom of the closed one-string partition function

$$
Z_{1}(\beta, \mu)=\frac{\beta}{2 \pi l_{s}} \int_{0}^{\infty} \frac{d \tau_{2}}{\tau_{2}^{2}} \int d \tau_{1} \exp \left(-\frac{\beta^{2}}{2 \pi \alpha^{\prime} \tau_{2}}\right) z_{l c}^{(0,0)}\left(\tau, \frac{\mu \beta}{\tau_{2}}\right)
$$

is now determined with the help of the S-modular transformation (3.16). The integrand of (3.21) behaves as

$$
\frac{1}{\tau_{2}^{2}} e^{-\frac{\beta^{2}}{2 \pi \alpha^{\prime} \tau_{2}}} z_{l c}^{(0,0)}(\tau, \mu) \stackrel{\tau_{2} \rightarrow 0}{\longrightarrow} \frac{1}{\tau_{2}^{2}} e^{-\frac{\beta^{2}}{2 \pi \alpha^{\prime} \tau_{2}}}\left(1-e^{\left.-\frac{\mu \beta}{|\tau|}\right)^{-\frac{d-2}{2}}} e^{-2 \pi(d-2) \frac{\tau_{2}}{|\tau|^{2}} \gamma_{0}(\mu \beta)} .\right.
$$

Ignoring for the moment the fermionic degrees of freedom and judging only by (3.21), the Hagedorn phase transition occurs again at the point where $Z_{1}(\beta, \mu)$ becomes divergent

$$
-\frac{\beta_{H}^{2}}{2 \pi \alpha^{\prime}}-2 \pi(d-2) \gamma_{0}\left(\mu \beta_{H}\right)=0
$$

Note that since the mass parameter $m$ is temperature dependent $m=\mu \beta / \tau_{2}$, the finite temperature behavior of strings in the pp-wave background is fundamentally different than that of strings in flat space. We will come back to address this issue at length in Section 4.

\subsection{Fermions}

Let us briefly recall the thermal partition function of closed superstrings in flat space. The contribution of the fermionic (physical) degrees of freedom to the light-cone partition function

$$
z_{l c}^{(1 / 2,0)}(\tau)=\left(|q|^{\frac{1}{12}} \prod_{n=1}^{\infty}\left|1+q^{n}\right|^{2}\right)^{d-2}
$$


can be rewritten using Jacobi's triple product formula in terms of the $\Theta$-functions:

$$
\prod_{n=0}^{\infty} q^{\frac{1}{24}}\left(1+q^{n}\right)=\eta(\tau)^{-\frac{1}{2}}\left[\prod_{n=0}^{\infty}\left(1+q^{n+1}\right)^{2}\left(1-q^{n}\right)\right]^{\frac{1}{2}}=\left(\Theta_{2}(0, \tau) \eta(\tau)^{-1}\right)^{1 / 2}
$$

For type II superstrings $(d-2=8)$ the one string partition function is

$$
Z_{1}^{(1 / 2,0)}(\beta)=\frac{\beta}{2 \pi l_{s}} \int_{0}^{\infty} \frac{d \tau_{2}}{\tau_{2}^{2}} \int d \tau_{1} \exp \left(-\frac{\beta^{2}}{2 \pi \alpha^{\prime} \tau_{2}}\right) 2^{-16}\left|\Theta_{2}(0, \tau) \eta(\tau)^{-3}\right|^{8}
$$

Using some $\Theta$-function algebra: $\Theta_{2}(0, \tau) \Theta_{3}(0, \tau) \Theta_{4}(0, \tau)=\eta(\tau)^{3}$ and $\Theta_{3}(0, \tau) \Theta_{4}(0, \tau)=\Theta_{4}(0,2 \tau)$ the one-string partition function can be cast in a more concise form

$$
Z_{1}^{(1 / 2,0)}(\beta)=\beta \int_{0}^{\infty} \frac{d \tau_{2}}{\tau_{2}^{2}} \int d \tau_{1} \exp \left(-\frac{\beta^{2}}{2 \pi \alpha^{\prime} \tau_{2}}\right) 2^{-16}\left|\Theta_{4}(0,2 \tau)\right|^{-16}
$$

Next, by invoking the modular properties of $\Theta_{4}$

$$
\Theta_{4}(0, \tau)=(-i \tau)^{-\frac{1}{2}} \Theta_{2}\left(0,-\frac{1}{\tau}\right)
$$

one obtains the ultraviolet behavior of the integrand in (3.27):

$$
\tau_{2}^{2} e^{-\frac{\beta^{2}}{2 \pi \alpha^{\prime} \tau_{2}}} e^{\frac{2 \pi}{\tau_{2}}}
$$

Since near $\tau_{2} \rightarrow 0$ the integrand is convergent, the type II superstrings in flat space background can undergo a phase transition at $T_{H}=1 /\left(2 \pi l_{s}\right)$.

In the plane wave background the transverse fermions are massive and this prevents us from using the triple product formula or the $\Theta$-function technology. However, the fermionic partition function is still a modular form, and we can still perform an $S$-transformation in order to read-off its asymptotics for $\tau_{2} \rightarrow 0$.

The light-cone gauge-fixed action of the fermionic degrees of freedom for type IIB GS superstring in the plane wave background takes the form ${ }^{2}$

$$
\mathcal{S}=\int d^{2} z \sum_{a=1}^{8}\left(i S^{1, a} \partial_{z} S^{1, a}+i S^{2, a} \partial_{\bar{z}} S^{2, a}+2 m S^{1, a} S^{2, a}\right) .
$$

Since we are interested in evaluating the thermal partition function, we impose periodicity in the $\sigma$-direction and antiperiodicity in the world-sheet time direction. Therefore we make the identifications $S^{a}\left(\xi_{1}+1, \xi_{2}\right)=S^{a}\left(\xi_{1}, \xi_{2}\right), S^{a}\left(\xi_{1}, \xi_{2}+1\right)=-S^{a}\left(\xi_{1}, \xi_{2}\right), z=\xi_{1}+\tau \xi_{2}$, where as explained before $\tau=\lambda+i \frac{\beta}{2 \pi \alpha^{\prime} p^{+}}$. In the new coordinates the action becomes

$$
\mathcal{S}=\int d \xi_{1} d \xi_{2}\left(i S^{1, a}\left(-\bar{\tau} \partial_{1}+\partial_{2}\right) S^{1, a}+i S^{2, a}\left(\tau \partial_{1}-\partial_{2}\right) S^{2, a}+2 m S^{1, a} S^{2, a}\right)
$$

\footnotetext{
${ }^{2} \mathrm{~A}$ few manipulations are in order to bring the fermionic action in this form. One first redefines the Weyl complex space-time fermions of [7] by conveniently absorbing a factor of $\sqrt{p^{+}}: \theta^{\alpha}=\frac{S_{1}^{\alpha}+i S_{2}^{\alpha}}{\sqrt{2 p^{+}}}, \alpha=1 . .8$ and after evaluating the matrix $\Pi=\gamma^{1} \gamma^{2} \gamma^{3} \gamma^{4}$ present in the fermionic mass term $m p^{+} \bar{\theta} \Pi \theta$, a trivial re-shuffling of the variables $S^{\alpha}$ leads to the action presented here.
} 
Substituting the appropriate Fourier decomposition of the fermionic degrees of freedom

$$
S^{a}\left(\xi_{1}, \xi_{2}\right)=\sum_{n_{1}, n_{2} \in \mathbf{Z}} S_{n_{1}, n_{2}}^{a} e^{i 2 \pi n_{1} \xi_{1}} e^{i \pi\left(2 n_{2}+1\right) \xi_{2}}
$$

into the the path integral expression of the partition function we obtain

$$
\begin{aligned}
z_{l c}^{(0,1 / 2)}(\tau, m) & =\int \mathcal{D} S^{1} \mathcal{D} S^{2} e^{-\mathcal{S}\left[S^{1}, S^{2}\right]} \\
& =\left[\prod_{n_{1}, n_{2} \in \mathbf{Z}} \tau_{2}\left(\left(\frac{2 \pi}{4 \tau_{2}}\right)^{2}\left|n_{1} \tau+\frac{2 n_{2}+1}{2}\right|^{2}+m^{2}\right)\right]^{8} \\
& =\exp \left[16 \pi \tau_{2}\left(\frac{m}{2}+\sum_{n=1}^{\infty} \sqrt{n^{2}+m^{2}}\right)\right]\left\{\prod_{n \in \mathbf{Z}}\left(1+\exp \left[2 \pi\left(-\tau_{2} \sqrt{n^{2}+m^{2}}+i \tau_{1} n\right)\right]\right)\right\}^{8}
\end{aligned}
$$

where in the last step we used a result of [15].

Under the modular $S$-transformation, a function antiperiodic in the $\xi_{2}$ direction becomes antiperiodic in the $\xi_{1}$ direction. The partition function for fermions obeying this new boundary conditions is

$$
\begin{aligned}
z_{l c}^{(1 / 2,0)}(\tau, m) & =\left[\prod_{n_{1}, n_{2} \in \mathbf{Z}} \tau_{2}\left(\left(\frac{2 \pi}{4 \tau_{2}}\right)^{2}\left|\frac{2 n_{1}+1}{2} \tau+n_{2}\right|^{2}+m^{2}\right)\right]^{8} \\
& =\exp \left[16 \pi \tau_{2} \sum_{n=1 / 2}^{\infty} \sqrt{n^{2}+m^{2}}\right]\left\{\prod_{n \in \mathbf{Z}+1 / 2}\left(1-\exp \left[2 \pi\left(-\tau_{2} \sqrt{n^{2}+m^{2}}+i \tau_{1} n\right)\right]\right)\right\}^{8} .
\end{aligned}
$$

The Casimir energy for half-integer modes can be computed using $\zeta$-function regularization

$$
\begin{aligned}
\gamma_{1 / 2}(m) & =\sum_{n=1 / 2}^{\infty} \sqrt{n^{2}+m^{2}}=\frac{1}{2} \sum_{n=1}^{\infty} \sqrt{(2 n+1)^{2}+4 m^{2}}=\frac{1}{2} \sum_{n=1}^{\infty} \sqrt{n^{2}+4 m^{2}}-\sum_{n=1}^{\infty} \sqrt{n^{2}+m^{2}} \\
& =\frac{1}{2}\left[\gamma_{0}(2 m)-m\right]-\left[\gamma_{0}(m)-\frac{m}{2}\right] \\
& =\frac{1}{24}-\frac{1}{2} m^{2} \ln \left(\pi e^{-\gamma}\right)+\sum_{n=2}^{\infty}(-1)^{n}\left(2^{2 n-1}-1\right) \frac{\Gamma\left(n-\frac{1}{2}\right)}{n ! \Gamma\left(-\frac{1}{2}\right)} \zeta(2 n-1) m^{2 n}
\end{aligned}
$$

where in the first line we rewrite the sum over odd integers as the the sum over all minus even integers; in the second line we use the $\zeta$-function regularization introduced in (3.18). As we expected, the modular $S$-transformation relates the two partition functions

$$
z_{l c}^{(1 / 2,0)}(1 / \tau, \mu /|\tau|)=z_{l c}^{(0,1 / 2)}(\tau, \mu) .
$$

Once again, recall that $m$ is not a constant: $m=\mu \beta / \tau_{2}$. We can now easily extract the high-energy behavior of the fermionic light-cone partition function:

$$
z_{l c}^{(0,1 / 2)}(\tau, \mu) \stackrel{\tau_{2} \rightarrow 0}{\longrightarrow} e^{16 \pi \frac{\tau_{2}}{|\tau|^{2}} \gamma_{1 / 2}(\mu \beta)} .
$$


The two modular forms we have introduced in (3.33) and (3.35) are also generalization to the complex plane of the modular forms $f_{2}^{(m)}\left(e^{-2 \pi \tau_{2}}\right)$ and respectively $f_{4}^{(m)}\left(e^{-2 \pi \tau_{2}}\right)$ of [13] with the distinguishing property that they are not holomorphic functions of $\tau$.

\section{Hagedorn temperature of IIB strings in the pp-wave background}

We have by now computed all the ingredients that go into the partition function. In a sense we can now simply return to the light-cone string partition function already given by (3.3). However, after the detour of the path integral computation we have gained knowledge of the modular properties of (3.3), and thus paved the road to find out the high energy behavior of the partition function. The one-string partition function is

$$
Z_{1}(\beta, \mu)=\frac{\beta}{2 \pi l_{s}} \int_{0}^{\infty} \frac{d \tau_{2}}{\tau_{2}^{2}} \int d \tau_{1} \exp \left(-\frac{\beta^{2}}{2 \pi \alpha^{\prime} \tau_{2}}\right) z_{l c}^{(0,0)}\left(\tau, \frac{\mu \beta}{\tau_{2}}\right) z_{l c}^{(0,1 / 2)}\left(\tau, \frac{\mu \beta}{\tau_{2}}\right) .
$$

In terms of the one-string partition function the free energy is

$$
\begin{aligned}
F(\beta) & =-\frac{1}{2 \beta} \sum_{r=1}^{\infty}\left[1-(-1)^{r}\right] \frac{Z_{1}(\beta r)}{r} \\
& =-\frac{1}{4 \pi l_{s}} \int_{0}^{\infty} \frac{d \tau_{2}}{\tau_{2}^{2}} \int d \tau_{1} \sum_{r=1}^{\infty}\left[1-(-1)^{r}\right] e^{-\frac{\beta^{2} r^{2}}{2 \pi \alpha^{\prime} \tau_{2}}} z_{l c}^{(0,0)}\left(\tau, \frac{\mu \beta r}{\tau_{2}}\right) z_{l c}^{(0,1 / 2)}\left(\tau, \frac{\mu \beta r}{\tau_{2}}\right) .
\end{aligned}
$$

In flat space where the only dependence on the number of strings $r$ comes from $\exp \left(-\beta^{2} r^{2} /\left(2 \pi \alpha^{\prime} \tau_{2}\right)\right.$, one can explicitly perform the summation over $r$ and write the free energy as a combination of theta functions [12]. Given the more involved $r$ dependence in (4.2) we have not been able to find such a closed form of the free energy. However, it can still be argued that the main contribution arises from the one-string term. In this case the relevant integrand behaves as $(3.22,3.37)$ :

$$
\begin{aligned}
& \frac{1}{\tau_{2}^{2}} e^{-\frac{\beta^{2}}{2 \pi \alpha^{\prime} \tau_{2}}} z_{l c}^{(0,0)}\left(\tau, \frac{\mu \beta}{\tau_{2}}\right) z_{l c}^{(0,1 / 2)}\left(\tau, \frac{\mu \beta}{\tau_{2}}\right) \stackrel{\tau_{2} \rightarrow 0}{\longrightarrow} \\
& \frac{1}{\tau_{2}^{2}} e^{-\frac{\beta^{2}}{2 \pi \alpha^{\prime} \tau_{2}}}\left(1-e^{-\frac{\mu \beta}{\tau \tau}}\right)^{-4} e^{-16 \pi \frac{\tau_{2}}{|\tau|^{2}} \gamma_{0}(\mu \beta)} e^{16 \pi \frac{\tau_{2}}{|\tau|^{2}} \gamma_{1 / 2}(\mu \beta)}
\end{aligned}
$$

where we have used the S-modular transformation to arrive to the second line. Considering the region where the above expression diverges we find that the Hagedorn temperature for the GS IIB superstring in the plane wave background is determined by solving:

$$
-\frac{\beta_{H}^{2}}{2 \pi \alpha^{\prime}}-16 \pi \gamma_{0}\left(\mu \beta_{H}\right)+16 \pi \gamma_{1 / 2}\left(\mu \beta_{H}\right)=0
$$

and it depends parametrically on $\mu$. Taking $\mu=0$ in the above equation and making use of (3.18) and (3.35) we find that $\beta_{H}=2 \pi l_{s}$ which is nothing but the value corresponding to flat 
space. To further explore the behavior of the Hagedorn temperature we consider the limit of small $\mu$ in units of the string scale $l_{s}$, that is, $\mu l_{s} \ll 1$. Expanding (4.4) up to terms linear in $\mu$ and using the expressions $(3.18,3.35)$ we find

$$
T_{H} \approx \frac{1}{2 \pi l_{s}}+4 \mu, \quad \text { for } \quad \mu l_{\mathrm{s}} \ll 1,
$$

that is, the Hagedorn temperature is higher than its value for strings in flat space.

This result is very interesting and naturally leads us into the question of the behavior in the opposite limit, $\mu l_{s} \rightarrow \infty$. This limit is the more important due to the results of [8] where it has been established that in the context of the BMN limit it corresponds to the weakly coupled field theory. ${ }^{3}$ To better answer the question of the Hagedorn temperature for large $m$ we find it convenient to use an alternative expression for the Casimir energies (see the appendix for a derivation):

$$
\gamma_{0}(m)=\frac{m}{2}+\sum_{n=1}^{\infty} \sqrt{n^{2}+m^{2}}=\frac{m}{2}+\left[-\frac{m}{2}-\frac{-1+\gamma}{4} m^{2}-\frac{m}{\pi} \sum_{n=1}^{\infty} \frac{1}{n} K_{-1}(2 \pi n m)\right] .
$$

For the fermions we will simply use the identity described in (3.35) to relate their Casimir energy to that of bosons. In equation (4.4) we have that $\beta_{H}$ is proportional to the difference of the Casimir energies:

$$
\begin{aligned}
\frac{\beta_{H}^{2}}{2 \pi \alpha^{\prime}} & =16 \pi\left[\gamma_{1 / 2}\left(\mu \beta_{H}\right)-\gamma_{0}\left(\mu \beta_{H}\right)\right]=\frac{1}{2} \gamma_{0}\left(2 \mu \beta_{H}\right)-2 \gamma_{0}\left(\mu \beta_{H}\right) \\
& =\frac{\mu \beta_{H}}{\pi} \sum_{n=1}^{\infty} \frac{1}{n}\left[2 K_{-1}\left(2 \pi n \mu \beta_{H}\right)-K_{-1}\left(4 \pi n \mu \beta_{H}\right)\right] .
\end{aligned}
$$

As $\mu \rightarrow \infty$, the difference between the two Casimir energies approaches zero since all the Bessel functions in the infinite sum (4.6) vanish in the limit, and therefore the Hagedorn temperature is pushed toward infinity. Recalling that the Hagedorn temperature can be interpreted as the temperature where a winding state becomes tachyonic $[16]^{4}$, we can rephrase the above result as the disappearance of the tachyon associated with the Hagedorn temperature. In this form the result is similar to recent investigations of type $0 \mathrm{~B}[14,17]$, where the tachyon has been found to disappear in the $\mu \rightarrow \infty$ limit.

Having established the existence and some of the properties of the Hagedorn temperature we turn to the question of its nature. Namely, we would like to find out whether the Hagedorn temperature signals a maximal temperature or it simply points to the possibility of a phase transition. To answer this question we should consider the behavior of the free energy and the

\footnotetext{
${ }^{3}$ However, as mentioned in the introduction, we should be extremely careful conjecturing the relation of our results to the BMN limit since we simply do not work on that limit.

${ }^{4}$ From the path integral approach we obtain the free energy naturally expressed as an integral over the entire strip $\left(-1 / 2 \leq \tau_{1} \leq 1 / 2,0 \leq \tau_{2}<\infty\right)$. Using the fact that the integrand enjoys modular properties, the free energy can be rewritten as an integral over the fundamental domain only. The net effect of this manipulation is to add a trace over "winding" modes in the partition function. These modes can be interpreted as states of the string wrapping the temporal direction.
} 
specific heat as we approach the Hagedorn temperature from below. Working in the one-string approximation and assuming that the main contribution to the free energy (4.2) comes form the UV region and using (4.3), we find that, for fixed $\mu$, the free energy diverges near the Hagedorn temperature

$$
F \sim-\frac{1}{2 \pi l_{s}} \int_{0}^{1} d \tau_{2} \frac{1}{\tau_{2}^{2}} \exp \left(-\frac{1}{\tau_{2}}\left[\frac{\beta^{2}}{2 \pi \alpha^{\prime}}+16 \pi\left(\gamma_{0}(\mu \beta)-\gamma_{1 / 2}(\mu \beta)\right)\right]\right)
$$

Evaluating the integral we can estimate the behavior of the free energy near the Hagedorn temperature

$$
F(T) \sim C\left(\beta_{H}\right)^{-1} \frac{T_{H}}{T_{H}-T}+\text { analytic in }\left(\beta-\beta_{H}\right) .
$$

where $C\left(\beta_{H}\right)=\beta_{H}^{2} /\left(\pi \alpha^{\prime}\right)+16 \pi \beta_{H} \mu\left[\gamma_{0}^{\prime}\left(\mu \beta_{H}\right)-\gamma_{1 / 2}^{\prime}\left(\mu \beta_{H}\right)\right]$. Let us now turn to the specific heat which we write in terms of the free energy as

$$
c_{V}=\beta^{2} \frac{\partial^{2}}{\partial \beta^{2}} \ln Z=-\beta^{2} \frac{\partial^{2}}{\partial \beta^{2}} \beta F=-\beta^{2}\left[2 \frac{\partial}{\partial \beta} F+\beta \frac{\partial^{2}}{\partial \beta^{2}} F\right] .
$$

Using (4.9) one derives that the specific heat also blows up near the Hagedorn temperature. Therefore for IIB strings in RR plane wave background the Hagedorn temperature is limiting, and, at least from the point of view of a canonical ensemble analysis, there is no phase transition which the system undergoes at $T_{H}$. Curiously, in flat space only open strings seem to have a limiting Hagedorn temperature. At least to this level of scrutiny, IIB strings in the RR plane wave background behave as open strings.

\section{Note added:}

A better estimation of the free energy behavior (finite or divergent) at the Hagedorn temperature can be done by evaluating the $\tau_{1}$ integral, as pointed out by Brower et al. [18]. Using the saddle point approximation, the integral over $\tau_{1}$ of (4.3) yields

$$
\begin{aligned}
F & \sim-\frac{1}{2 \pi l_{s}} \int d \tau_{2} \frac{1}{\tau_{2}^{2}} e^{-\frac{\beta^{2}}{2 \pi \alpha^{\prime} \tau_{2}}} \int d \tau_{1} e^{\frac{\beta_{H}^{2}}{2 \pi \alpha^{\prime} \tau_{2}} \epsilon^{-\frac{\beta_{H}^{2} \tau_{1}^{2}}{2 \pi \alpha^{\prime} \tau_{2}^{3}}}} \\
& \sim-\frac{1}{\beta_{H}} \int d \tau_{2} \tau_{2}^{-1 / 2} e^{\frac{\beta_{H}^{2}-\beta^{2}}{2 \pi \alpha^{\prime} \tau_{2}}} \sim-c\left(\beta_{H}\right) \sqrt{\beta-\beta_{H}}+\text { regular }
\end{aligned}
$$

which should be compared with the rougher approximation used in (4.8), where the integral over $\tau_{1}$ was omitted.

Thus the free energy is, in the end, finite, and a phase transition may occur when the system reaches the Hagedorn temperature. Note however that the specific heat is infinite at $T_{H}$, and negative. 


\section{Acknowledgments}

We are grateful to G. D’Appollonio, R. Emparan, A. Hashimoto, J. Maldacena, N. Quiroz, J. Sonnenschein, C. Thorn and A. Tseytlin. We are grateful to B. Greene, K. Schalm and G. Shiu for comments on a previous version of the manuscript and for informing us of their related work. We are especially thankful to Igor Klebanov for a very illuminating discussion. The work of D.V. is supported by DOE grant DE-FG02-91ER40671.

\section{Appendix A}

For completeness we include a brief derivation of (4.6).

$$
\begin{aligned}
& \sum_{n=1}^{\infty} \sqrt{n^{2}+m^{2}}=\frac{1}{2}\left(\sum_{n \in \mathbf{Z}} \sqrt{n^{2}+m^{2}}-m\right)=\frac{1}{2}[F(0)-m] \\
& F(a)=\sum_{n \in \mathbf{Z}} \sqrt{(n+a)^{2}+m^{2}}
\end{aligned}
$$

Using the Poisson summation formula we can write

$$
F(a)=\sum_{k \in \mathbf{Z}} \exp (2 \pi i k a) \int_{-\infty}^{\infty} d y \exp (-2 \pi i k y) \sqrt{y^{2}+m^{2}}
$$

Next substitute

$$
\sqrt{y^{2}+m^{2}}=\frac{1}{\Gamma(-1 / 2)} \int_{0}^{\infty} d t t^{-3 / 2} \exp \left[-t\left(y^{2}+m^{2}\right)\right]
$$

into (A.3), perform the Gaussian integral over $y$ and arrive at

$$
F(a)=\frac{\sqrt{\pi}}{\Gamma(-1 / 2)} \sum_{k \in \mathbf{Z}} \exp (2 \pi i k a) \int_{0}^{\infty} d t t^{-2} \exp \left(-t m^{2}-\frac{k^{2} \pi^{2}}{t}\right) .
$$

The term $k=0$ in the sum leads to a divergent expression, proportional to $\Gamma(-1) / \Gamma(-1 / 2)$ which is further regulated by keeping only the finite part of $\Gamma(-1+\epsilon)$, with $\epsilon \rightarrow 0$.

After one more change of variable $v=t m /(\pi|k|)$, the other terms in (A.5) can be expressed in terms of modified Bessel functions, and we obtain

$$
F(a)=-\frac{-1+\gamma}{2} m^{2}-\sum_{k=1}^{\infty} \frac{2 m \cos (2 \pi k a)}{\pi k} K_{-1}(2 \pi k m) .
$$

Substituting this expression into (A.1) completes the derivation of (4.6). 


\section{References}

[1] J. Maldacena, "The large N limit of superconformal field theories and supergravity," Adv. Theor. Math. Phys. 2 (1998) 231 [hep-th/9711200].

S.S. Gubser, I.R. Klebanov and A.M. Polyakov, "Gauge theory correlators from non-critical string theory," Phys. Lett. B428 (1998) 105 [hep-th/9802109].

E. Witten, "Anti-de Sitter space and holography," Adv. Theor. Math. Phys. 2 (1998) 253 [hep-th/9802150].

[2] E. Witten, "Anti-de Sitter space, thermal phase transition, and confinement in gauge theories," Adv. Theor. Math. Phys. 2 (1998) 505 [arXiv:hep-th/9803131].

[3] S. S. Gubser, I. R. Klebanov and A. W. Peet, "Entropy and Temperature of Black 3Branes," Phys. Rev. D 54 (1996) 3915 [arXiv:hep-th/9602135].

S. S. Gubser, I. R. Klebanov and A. A. Tseytlin, "Coupling constant dependence in the thermodynamics of $\mathrm{N}=4$ supersymmetric Yang-Mills theory," Nucl. Phys. B 534 (1998) 202 [arXiv:hep-th/9805156].

[4] D. Berenstein, J. M. Maldacena and H. Nastase, "Strings in flat space and pp waves from N = 4 super Yang Mills," JHEP 0204 (2002) 013 [arXiv:hep-th/0202021].

[5] M. Blau, J. Figueroa-O'Farrill and G. Papadopoulos, "Penrose limits, supergravity and brane dynamics," arXiv:hep-th/0202111.

M. Blau, J. Figueroa-O'Farrill, C. Hull and G. Papadopoulos, "A new maximally supersymmetric background of IIB superstring theory," JHEP 0201 (2002) 047 [arXiv:hepth/0110242].

[6] R. R. Metsaev, "Type IIB Green-Schwarz superstring in plane wave Ramond-Ramond background," Nucl. Phys. B 625 (2002) 70 [arXiv:hep-th/0112044].

[7] R. R. Metsaev and A. A. Tseytlin, "Exactly solvable model of superstring in plane wave Ramond-Ramond background," Phys. Rev. D 65 (2002) 126004 [arXiv:hep-th/0202109]. J.G. Russo and A.A. Tseytlin "On solvable models of type $2 \mathrm{~B}$ superstring in NS-NS and R-R wave backgrounds", JHEP 0204 (2002) 021 [arXiv: hep-th/0202179].

[8] C. Kristjansen, J. Plefka, G. W. Semenoff and M. Staudacher, "A new double-scaling limit of $\mathrm{N}=4$ super Yang-Mills theory and PP-wave strings," arXiv:hep-th/0205033.

D. Berenstein and H. Nastase, "On lightcone string field theory from super Yang-Mills and holography," arXiv:hep-th/0205048.

D. J. Gross, A. Mikhailov and R. Roiban, "Operators with large R charge in N = 4 YangMills theory," arXiv:hep-th/0205066.

N. R. Constable, D. Z. Freedman, M. Headrick, S. Minwalla, L. Motl, A. Postnikov and W. Skiba, "PP-wave string interactions from perturbative Yang-Mills theory," JHEP 0207 (2002) 017 [arXiv:hep-th/0205089].

A. Santambrogio and D. Zanon, "Exact anomalous dimensions of $\mathrm{N}=4$ Yang-Mills operators with large R charge," arXiv:hep-th/0206079. 
[9] I. R. Klebanov, M. Spradlin and A. Volovich, "New effects in gauge theory from pp-wave superstrings," arXiv:hep-th/0206221.

[10] L. A. Pando Zayas and J. Sonnenschein, "On Penrose limits and gauge theories," JHEP 0205 (2002) 010 [arXiv:hep-th/0202186].

[11] E. Alvarez, "Strings At Finite Temperature," Nucl. Phys. B 269 (1986) 596.

[12] E. Alvarez and M. A. Osorio, "Superstrings At Finite Temperature," Phys. Rev. D 36 (1987) 1175.

[13] O. Bergman, M. R. Gaberdiel and M. B. Green, "D-brane interactions in type IIB planewave background," arXiv:hep-th/0205183.

[14] T. Takayanagi, "Modular invariance of strings on pp-waves with RR-flux," arXiv:hepth/0206010.

[15] H. Saleur and C. Itzykson, "Two-Dimensional Field Theories Close to Criticality," Journal of Stat. Phys. 48 (1987) 449.

[16] J. Polchinski, "Evaluation Of The One Loop String Path Integral," Commun. Math. Phys. 104 (1986) 37.

J.J. Atick, E. Witten, "The Hagedorn Transition and the Number of Degrees of Freedom of String Theory", Nucl.Phys. B310 (1988) 291.

[17] F. Bigazzi, A. L. Cotrone, L. Girardello and A. Zaffaroni, "pp-wave and nonsupersymmetric gauge theory," arXiv:hep-th/0205296.

[18] R. C. Brower, D. A. Lowe and C. -I. Tan, "Hagedorn transition for strings on pp-waves and tori with chemical potentials," arXiv: hep-th/0211201. 